\title{
Spectral evidence for a powerful compact jet from XTE J1118+480
}

\author{
R. P. Fender ${ }^{1}$, R. M. Hjellming ${ }^{2}$, R. P. J. Tilanus ${ }^{3}$, G. G. Pooley ${ }^{4}$, J. R. Deane ${ }^{5}$, \\ R. N. Ogley ${ }^{6}$, R. E. Spencer ${ }^{7}$ \\ 1 Astronomical Institute 'Anton Pannekoek', University of Amsterdam, and Center for High Energy Astrophysics, Kruislaan 403, \\ 1098 SJ, Amsterdam, The Netherlands rpf@astro.uva.nl \\ 2 National Radio Astronomy Observatory, Socorro, NM 87801, USA \\ 3 Joint Astronomy Centre, 660 N. A'ohoku Pl., Hilo, Hawaii, USA \\ 4 Mullard Radio Astronomy Observatory, Cavendish Laboratory, Madingley Road, Cambridge CB3 OHE ggp1@cam.ac.uk \\ 5 Institute for Astronomy, University of Hawaii, 2680 Woodlawn Drive, Honolulu, HI 96822, USA \\ ${ }^{6}$ Service d'Astrophysique, CEA Saclay, F-91191 Gif sur Yvette, Cedex, France \\ 7 University of Manchester, Nuffield Radio Astronomy Laboratories, Jodrell Bank, Cheshire, SK11 9DL
}

9 November 2018

\begin{abstract}
We present observations of the X-ray transient XTE J1118+480 during its Low/Hard X-ray state outburst in 2000, at radio and sub-millimetre wavelengths with the VLA, Ryle Telescope, MERLIN and JCMT. The high-resolution MERLIN observations reveal all the radio emission (at $5 \mathrm{GHz}$ ) to come from a compact core with physical dimensions smaller than $65 \mathrm{~d}(\mathrm{kpc}) \mathrm{AU}$. The combined radio data reveal a persistent and inverted radio spectrum, with spectral index $\sim+0.5$. The source is also detected at $350 \mathrm{GHz}$, on an extrapolation of the radio spectrum. Flat or inverted radio spectra are now known to be typical of the Low/Hard X-ray state, and are believed to arise in synchrotron emission from a partially self-absorbed jet. Comparison of the radio and sub-millimetre data with reported near-infrared observations suggest that the synchrotron emission from the jet extends to the near-infrared, or possibly even optical regimes. In this case the ratio of jet power to total X-ray luminosity is likely to be $P_{\mathrm{J}} / L_{\mathrm{X}}>>0.01$, depending on the radiative efficiency and relativistic Doppler factor of the jet. Based on these arguments we conclude that during the period of our observations XTE J1118+480 was producing a powerful outflow which extracted a large fraction of the total accretion power.
\end{abstract}

Key words:

binaries: close - stars: individual: XTE J1118+480 - radio continuum: stars - ISM:jets and outflows

\section{INTRODUCTION}

XTE J1118+480 is a new transient X-ray source, discovered in soft $(\leq 12 \mathrm{keV}) \mathrm{X}$-rays by the all-sky monitor (ASM) onboard the Rossi X-ray Timing Explorer (RXTE) in 2000 March (Remillard et al. 2000). Within days optical (Uemura, Kato \& Yamaoka 2000a), radio (Pooley \& Waldram 2000) and hard X-ray (Wilson \& McCollough 2000) counterparts to the new transient were established. Uemura et al. (2000b) report in detail the discovery and subsequent photometric observations of the optical counterpart. Garcia et al. (2000) reported strong $\mathrm{H} \alpha$ emission, and established that the interstellar extinction towards the source was very low. This fact allowed for the first time EUV observations of an X-ray transient (Hynes et al. 2000). Patterson (2000) further reported rapid and erratic variability in the optical flux from the system. Merloni, Di Matteo \& Fabian (2000) have modelled the rapid optical variability as synchrotron emission from magnetic flares in a corona above the accretion disc.

The X-ray properties of XTE J1118+480, both in timing and spectra, indicate that the system is a black hole (candidate) in the Low/Hard X-ray state (Revnivtsev, Sunyaev \& Borozdin 2000; Wood et al. 2000), characterised by strong low-frequency variability and a hard power-law component extending to $\gtrsim 100 \mathrm{keV}$ which dominates any contribution in X-rays from an accretion disc. The radio spectrum of the system is persistently inverted in the range $1-22 \mathrm{GHz}$ 
(Dhawan et al. 2000; Hynes et al. 2000) with a spectral in$\operatorname{dex}\left(\alpha\right.$, where $\left.S_{\nu} \propto \nu^{\alpha}\right)$ of about +0.5 . Such flat or inverted radio spectra have been established as a ubiquitous observational characteristic of the Low/Hard X-ray state in both peristent and transient black hole X-ray binaries (Fender 2000a,b).

\section{OBSERVATIONS}

During a 100-day period between MJD 51620 - 51720 XTE J1118+480 exhibited a steady level of X-ray and radio emission. The observations reported below were obtained during this steady phase. More recently the source has declined rapidly again (since around MJD 51745).

\subsection{VLA}

Regular monitoring of XTE J1118+480 has been undertaken by the Very Large Array (VLA) throughout the 2000 outburst. During the interval MJD 51620 - 51720, the mean flux densities at $1.4,8.4$ and $22.5 \mathrm{GHz}$ were $2.6 \pm 0.4,6.5 \pm 0.7$ and $9.3 \pm 1.2$ mJy respectively, where uncertainties reflect the statistical deviation or the sample (intrinsic source variability and/or calibration errors) rather than the significance of the detections.

\subsection{RT}

The Ryle Telescope (RT) was used to monitor the flux density at $15.2 \mathrm{GHz}$, using techniques similar to those described in Pooley \& Fender (1997). The phase calibrator used was $\mathrm{J} 1110+440$, and the flux-density scale established by observations of $3 \mathrm{C} 48$ and $3 \mathrm{C} 286$. The mean flux density measured by the RT over the period under discussion was $9.0 \pm 1.0 \mathrm{mJy}$.

\subsection{MERLIN}

XTE J1118+480 was observed by MERLIN on 2000 Apr 5 1650-2053 UT, in standard continuum observing mode at $4.994 \mathrm{GHz}$ with a $15 \mathrm{MHz}$ bandwidth, and primary and secondary flux calibrators 3C286 and OQ208. To phasecalibrate the target source a 6 minute cycle nodding between the target and phase calibrator $1107+485$ was used. Even with the short integration time and therefore incomplete $u v$ coverage, a fully calibrated image of $1118+480$ was obtained (Fig 1). The image has a $1 \sigma$ noise level of $248 \mu \mathrm{Jy} / \mathrm{beam}$ with a synthesised beam of $65 \times 35$ mas at 90 degrees from north. XTE J1118+480 is an unresolved point source at coordinates RA(2000) $111810.794 \operatorname{Dec}(2000)+480212.42$ with a flux density of $5.16 \mathrm{mJy}$. The MERLIN run was simultaneous with an observation with the RT, which measured a flux density of $8.7 \pm 0.3 \mathrm{mJy}$ at $15 \mathrm{GHz}$, confirming the inverted spectrum $(\alpha=+0.5)$.

\subsection{JCMT}

XTE J1118+480 was observed at $350 \mathrm{GHz}(850 \mu \mathrm{m})$ with the SCUBA instrument on the James Clerk Maxwell Telescope (JCMT) on 2000 May 30 and 31. The atmospheric

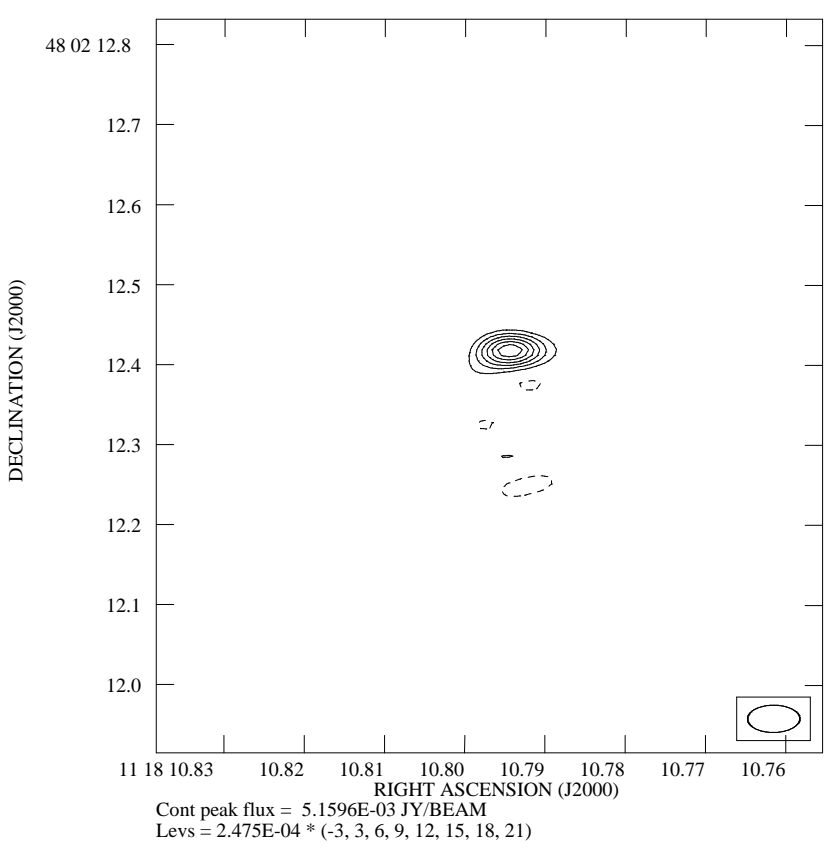

Figure 1. $5 \mathrm{GHz}$ MERLIN image of XTE J1118+480, revealing a compact unresolved core. Comparison with RT and VLA monitoring indicates that all the radio emission arises within this core. The synthesised beam, $65 \times 35$ mas, is indicated in the lower right-hand corner.

optical depth at $850 \mu \mathrm{m}$ was variable but around 0.4 during both observations (based on skydips and measurements from the nearby Caltech Submillimetre Observatory). Flux calibration was performed using Uranus and CIT6. XTE $\mathrm{J} 1118+480$ is clearly detected on both nights (with no variability at $>20 \%$ level between the two runs). The mean flux density from the two nights' observations is $41 \pm 4 \mathrm{mJy}$, the error being dominated by the uncertainty in the flux conversion factor.

Another observation of the source at $350 \mathrm{GHz}$ was performed on 2000 September 9, at which time only a $(3 \sigma)$ upper limit of $21 \mathrm{mJy}$ was obtained. Given the decay in radio emission from XTE J1118+480 by this time this supports the association of the submillimetre source with the X-ray transient.

\section{DISCUSSION}

In Fender (2000a,b) it has been shown that all X-ray binaries in the Low/Hard X-ray state produce a flat or inverted radio spectrum (i.e. $\alpha \geq 0$ ), which is likely to extend spectrally to the millimetre or infrared regimes. This spectral component is likely to arise in a partially self-absorbed synchrotron emission from a relativistic outflow or jet from each system. The power into this outflow appears to be a significant $(\geq 5 \%)$ and approximately fixed fraction of the accretion luminosity. In the following we discuss the observations of XTE J1118+480 in the context of such a model. 


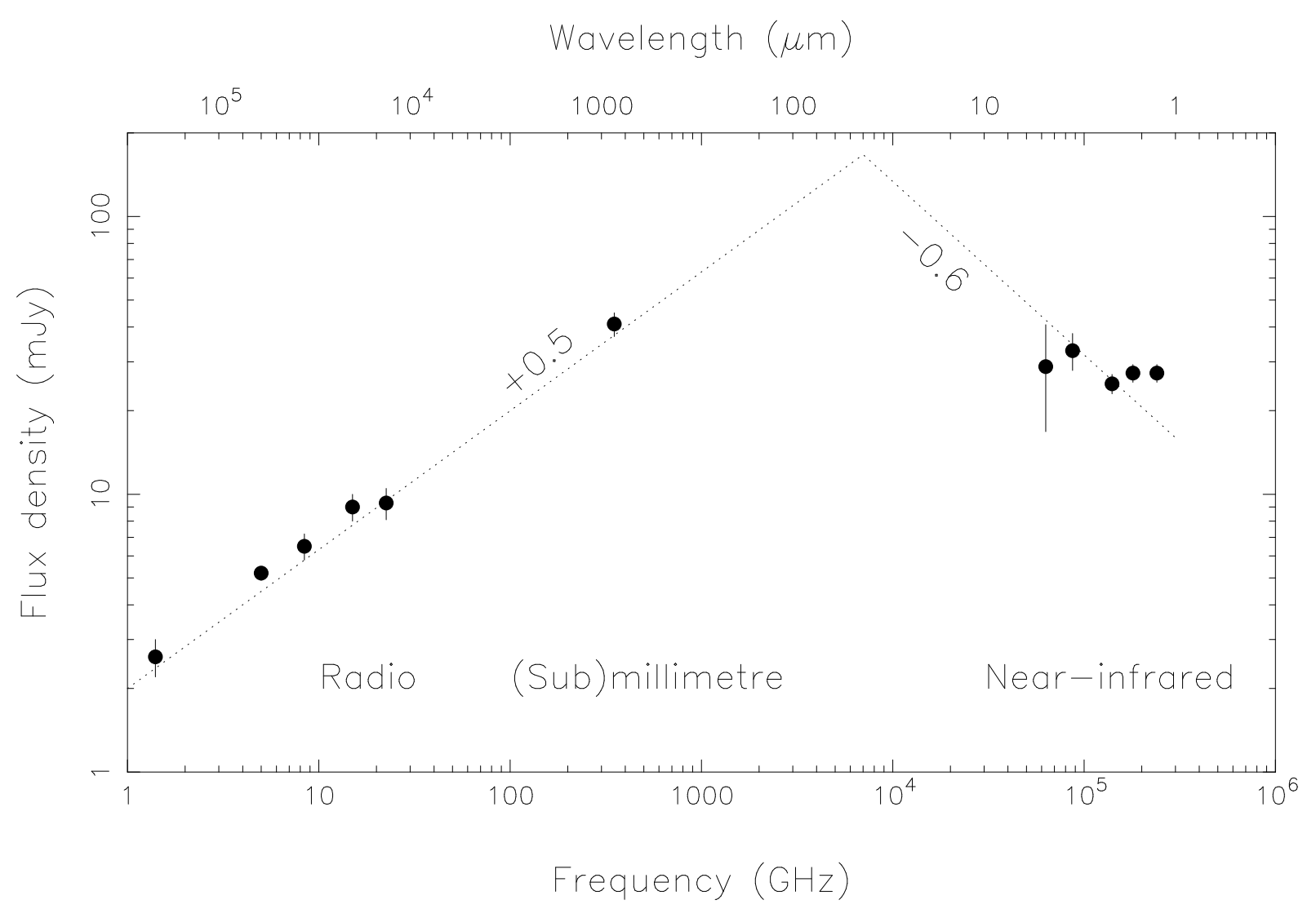

Figure 2. Radio - Sub-mm - Near-infrared broadband spectrum of XTE J1118+480 during the extended, steady, period in the Low/Hard X-ray state (MJD 51620-51720). Note the inverted radio spectrum which extrapolates directly to the sub-mm measurement at $350 \mathrm{GHz}$; this component is likely to be synchrotron emission from a compact, partially self-absorbed jet. The near-infrared data are from Hynes et al. (2000); there is strong evidence for an additional contribution at longer wavelengths (see text and Fig 3), which may be the optically thin extension of the jet spectrum. Also indicated on the figure is a double power-law corresponding simplistically to self-absorbed and optically thin regimes in the jet.

\subsection{Compact core}

The flux density of the unresolved component imaged with MERLIN is consistent with the radio spectrum measured by the VLA and RT, therefore we can be confident that all the radio emission from XTE $\mathrm{J} 1118+480$ arises within a region smaller than the MERLIN beam. We note that the (onesided) radio jet from Cyg X-1 in the Low/Hard state has an angular extent of $\sim 15$ mas at $8 \mathrm{GHz}$ (Stirling, Spencer \& Garrett 1998; Stirling et al. 2000), at a distance of $\sim 2$ kpc. Given a comparable GHz flux density we might expect a similar angular extent from XTE J1118+480, so the $35 \times 65$ mas beam probably failed to resolve the core by less than a factor of 10 (and the system may have been resolveable with the VLBA, depending on orientation).

\subsection{Broadband spectrum}

Fig 2 plots the broadband spectrum (SED) of XTE J1118+480, from radio to near-infrared wavelengths, during the $\sim 100$-day steady period in the Low/Hard X-ray state (see also Hynes et al. 2000 for a more extensive SED, but without the sub-millimetre datum). Within uncertainties dominated by the non-simultaneity of the observations, the radio data correspond to a power-law of spectral index $\sim+0.5$. The JCMT measurement sits exactly on an extrap- olation of this power-law to the sub-millimetre regime. We therefore consider it most likely that emission at $350 \mathrm{GHz}$ is an extension of the inverted spectral component from the radio. Furthermore, the significant decrease in the $350 \mathrm{GHz}$ flux density when the source was reobserved in 2000 September, by which point the radio flux density had also dropped dramatically, supports this interpretation.

It is unlikely that our detection of the system at 350 $\mathrm{GHz}$ is exactly at the high-frequency break of the inverted spectral component, however comparison with the nearinfrared fluxes reported by Hynes et al. (2000) indicates that some break, probably to an optically thin spectrum, occurs before $10^{14} \mathrm{~Hz}$ (Fig 2). Naively assuming that the inverted spectral component continues with a spectral index of +0.5 and then breaks at one point to an optically thin spectrum with $\alpha=-0.6$ (a typical value) which connects with the lowest-frequency near-infrared point (see below), the break will occur around $\sim 7 \times 10^{12} \mathrm{~Hz}(40 \mu \mathrm{m})$, with a peak flux density of about $150 \mathrm{mJy}$. This crude 'fit' to the radio-mminfrared data is plotted in Fig 2.

Three sets of near-infrared observations have been reported, in Hynes et al. (2000) and Taranova \& Seranova (2000); these are plotted in Fig 3. Since the extinction to the source is very low $(\mathrm{E}(\mathrm{B}-\mathrm{V})=0.013-$ Hynes et al. 2000), it is not necessary to deredden the infrared data. Bearing in 


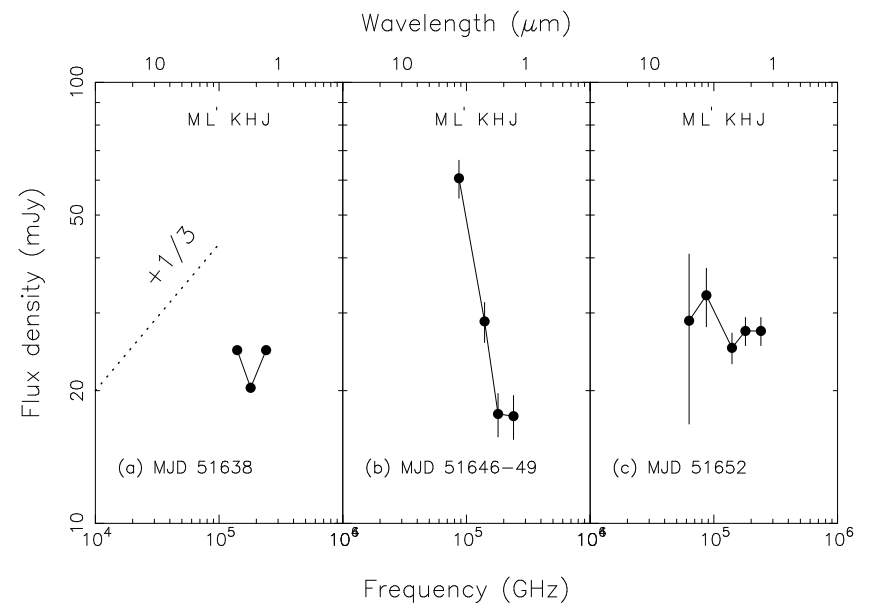

Figure 3. Three sets of reported near-infrared observations of XTE J1118+480, obtained during the period of relatively steady $\mathrm{X}$-ray state. Thermal emission from e.g. an accretion disc would be expected to have a positive spectral index (ie. rising with frequency) in the range $0.3-2.0$; in panel (a) a spectral index of $+1 / 3$ is indicated. These data clearly indicate an excess above any such thermal emission. Data from Hynes et al. (2000; panels (a) and (c)) and Taranova \& Shenavrin (2000; panel (b)).

mind that an accretion disc should have a thermal spectrum with spectral index in the range $1 / 3 \lesssim \alpha \lesssim 2$, it appears that there is excess infrared emission at the longest wavelengths $(\lambda \geq 2 \mu \mathrm{m}$, ie. in the K-band and beyond). Hynes et al. (2000) have already noted that another source of nearinfrared flux (beyond an accretion disc) is present. Since the sub-mm detection clearly demonstrates that the inverted spectral component does not cut off in the radio band, it seems plausible to connect it to the excess emission in the near-infrared. The reader is reminded that there is already very strong evidence for synchrotron emission from the radio through the millimetre to the near-infrared from the black hole system GRS 1915+105 when in hard X-ray states (e.g. Fender \& Pooley 1998, 2000 and references therein), and that Fender (2000b) presents observational evidence that the synchrotron spectrum may extend to the near-infrared or even optical regimes in all black hole X-ray binaries which are in the Low/Hard X-ray state.

\section{$3.3 \quad$ Energetics}

The integrated $1-350 \mathrm{GHz}$ luminosity of the invertedspectrum component is $L_{\mathrm{J}}=10^{31}(d / \mathrm{kpc})^{2} \mathrm{erg} \mathrm{s}^{-1}$. When in the Low/Hard X-ray state, Cyg X-1 has a typical XTE ASM count rate of $20 \mathrm{ct} / \mathrm{sec}$; it is assumed to lie at a distance of $\sim 2 \mathrm{kpc}$ and has an integrated X-ray luminosity (dominated by the power-law component) of $3 \times 10^{37} \mathrm{erg} \mathrm{s}^{-1}$ (Di Salvo et al. 2000). Therefore, under the (reasonable) assumption that all X-ray binaries in the Low/Hard state have comparable spectra, we scale from Cyg X-1 to derive the relation $L_{\mathrm{X}, \mathrm{LHS}} \sim 4 \times 10^{35} R(\mathrm{~d} / \mathrm{kpc})^{2} \mathrm{erg} \mathrm{s}^{-1}$, where $R$ is the XTE/ASM 2-12 keV count rate. The mean XTE ASM count rate for XTE J1118+480 at the time of our observations was around 2 , so in this case $L_{\mathrm{X}} \sim 8 \times 10^{35}(\mathrm{~d} / \mathrm{kpc})^{2}$ erg s $\mathrm{s}^{-1}$. Broadband X-ray observations (McClintock et al. 2000) confirm this estimate of the $2-200 \mathrm{keV}$ luminosity to within a factor of two, validating the method (however those authors also note that the X-ray spectrum may peak at even higher energies in this source). Note that simply using measured soft X-ray fluxes from XTE J1118+480, such as those reported in Remillard et al. (2000) will underestimate the $\mathrm{X}$-ray luminosity, which normally peaks around $100 \mathrm{keV}$ in the hard state, but has been accurately measured for Cyg $\mathrm{X}-1$. From this, $L_{\mathrm{J}} / L_{\mathrm{X}} \sim 2 \times 10^{-5}$; this should be considered as a firm lower limit to the ratio of jet to X-ray luminosities (assuming the impossible case of a $100 \%$ radiatively efficient jet), unless the X-ray spectrum of XTE J1118+480 really turns out to be very different from that of Cyg X-1 at higher $(>200 \mathrm{keV})$ energies.

If the jet spectrum is something like the dual powerlaw 'fit' shown in Fig 2, then the integrated luminosity of the inverted spectral component is $8 \times 10^{32}(\mathrm{~d} / \mathrm{kpc})^{2} \mathrm{erg} \mathrm{s}^{-1}$. However the radiative luminosity is dominated by the extent of the high-frequency emission; regardless of the spectral form at lower frequencies, if the jet contributes $\sim 20$ mJy at $3 \times 10^{14} \mathrm{~Hz}$ then the integrated radiative luminosity $L_{\mathrm{J}} \geq 10^{34}(\mathrm{~d} / \mathrm{kpc})^{2}$ erg $\mathrm{s}^{-1}$. ie. $L_{\mathrm{J}} / L_{\mathrm{X}} \geq 0.01$. Even if the jet spectrum peaks at $350 \mathrm{GHz}$ and a power law (with $\alpha \sim-0.1$ ) connects directly from the submillimetre to the infrared regimes $L_{\mathrm{J}}$ is reduced from that of the 'fit' by less than $10 \%$. The total jet power can be estimated as $P_{\mathrm{J}} \sim L_{\mathrm{J}} \eta^{-1} F(\Gamma, i)$, where $\eta$ is the radiative efficiency of the jet and $F(\Gamma, i)=\Gamma \delta^{-3}$ is a correction for relativistic motion (Fender 2000b). Assuming $\eta=0.05$ (which seems reasonable - Fender 2000b and references therein), and $F(\Gamma, i)=1$ (ie. no significant relativistic correction), the jet power will be $\geq 20 \%$ of the integrated X-ray luminosity, and therefore a very significant factor indeed for the energetics of the system. How reasonable is the assumption $F(\Gamma, i) \sim 1$ ? Dubus et al. (2000) estimate the orbital inclination of the binary to be $30 \leq i \leq 70$ based on optical spectroscopy. For $\Gamma \leq 5$, $0.2 \leq F(\Gamma, i) \leq 200$; ie. the maximum we could be overestimating the jet power by is a factor of 5 , whereas we could be underestimating it by two orders of magnitude.

\subsection{Optical variability}

Merloni et al. (2000) argue that the rapid optical variability of XTE J1118+480, and in particular its correspondence with X-ray variability, indicates that the optical flux is generated in the inner regions of the accretion disc, and is of nonthermal origin. Rapid near-infrared variability in phase with X-ray variability has been directly observed from GRS 1915+105 (Eikenberry et al. 1998; Mirabel et al. 1998), and in this source there is little doubt that the infrared flux is associated with powerful relativistic ejections (Fender \& Pooley 1998, 2000; Dhawan, Mirabel \& Rodriguez 2000). In Cyg X-3 rapid near-infrared flares are also probably associated with a relativistic jet (Fender et al. 1996). Since we have argued that the excess near-infrared flux is associated with the jet emission, and rapid near-infrared variability seems often to be associated with jet sources, we suggest that the rapid optical flaring in XTE $\mathrm{J} 1118+480$ is also associated with the jet, probably being optically thin nonthermal synchrotron emission from the 'base' of the jet, very close to the accretion disc. This is similar to the model of Merloni et al. (2000), except that we envisage the sites of optical emission as being associated with a global structure which, at radio wavelengths at least, is resolved into a collimated outflow. 


\section{CONCLUSIONS}

We have presented radio and sub-mm observations of the X-ray transient XTE J1118+840, and combined them with published near-infrared data to present the broadband spectrum of the system from $1-10^{6} \mathrm{GHz}$ during a steady period of Low/Hard X-ray state emission. The radio spectrum is inverted, with $\alpha=+0.5$, consistent with flat/inverted spectra from partially self-absorbed jets being a generic feature of the Low/Hard X-ray state (Fender 2000a,b). Two lines of argument are presented to argue that this spectral component extends from the radio regime through to the near-infrared

- The detection of the system at $350 \mathrm{GHz}(850 \mu \mathrm{m})$ precisely on an extrapolation of the inverted radio spectrum. This clearly indicates that the inverted spectral component extends significantly beyond the radio band.

- Excess flux in reported near-infrared measurements. Three sets of reported near-infrared photometry of XTE $\mathrm{J} 1118+480$ all reveal evidence for excess, apparently nonthermal, infrared emission in about the K-band and beyond

Using these two observational facts, we argue that the inverted spectral component extends from the radio regime all the way to the near-infrared or optical regimes. If this is the case, then the ratio of jet power to X-ray luminosity is $P_{\mathrm{J}} / L_{\mathrm{X}} \sim 0.01 \eta^{-1}$, where $\eta$ is the radiative efficiency of the jet (see Fender 2000b). For discrete ejection events from GRS 1915+105 (which clearly have a flat/inverted spectrum extending from the radio, through the $\mathrm{mm}$, to the nearinfrared regime) the maximum radiative efficiency appears to be $\sim 0.05$ (Fender \& Pooley 2000); therefore the jet power is likely to be a major, if not perhaps dominant, power output channel for the system in this X-ray state.

It is interesting to think about the size scales associated with the jet; for a Blandford \& Königl (1979) jet the physical size associated with emission at a given frequency scales as $\nu^{-1}$. If me assume the jet to have similar dimensions to that of $\mathrm{Cyg} \mathrm{X}-1$, ie. $\sim 30 \mathrm{AU}$ at 8 GHz, then eminssion at 350 $\mathrm{GHz}$ will arise from a region of size scale $\lesssim 1 \mathrm{AU}$. If the inverted component does peak around $7 \times 10^{12} \mathrm{~Hz}$, this would correspond to a size scale of around $0.03 \mathrm{AU}$, or $5 \times 10^{11} \mathrm{~cm}$. The optically thin emission observed in the near-infrared will come from even smaller phyiscal scales, whose size would be best constrained by rapid variability.

XTE J1118+480 is not unique in showing evidence for jet emission extending to the near-infrared when in the 'canonical' Low/Hard X-ray state. Fender (2000b) argues that the synchrotron spectrum probably extends to the nearinfrared or optical regimes in all X-ray binaries in this state. Corbel \& Fender (2001) and Corbel et al. (2001) present further evidence for near-infrared synchrotron emission from GX 339-4 and XTE J1550-564 when in the Low/Hard X-ray state, a persistent and transient source respectively. Brocksopp et al. (2000) finds similarities in the optical properties of Low/Hard state transients, which may also be related to high-frequency synchrotron emission associated with the jet. However in most cases the sub-mm and far-infrared regimes, crucial for establishing the connection between the radio and near-infared emission, are not explored. Such observations, admittedly technically difficult, are vital for confirming our qualitative model and establishing that in the Low/Hard
X-ray state the jet can be a major channel for the output of accretion power from black holes. In a more theoretical study Markoff, Falcke \& Fender (2001) have applied a jet-dominated model to the broadband radio through $\mathrm{X}$ ray spectrum of XTE $\mathrm{J} 1118+480$, and found that even the power-law X-ray component may arise as in the jet, possibly as direct optically thin synchrotron emission. In this case more than $90 \%$ of the power output of the system is in the form of the jet. While ironically the model of Markoff et al. (2001) is unable to fit the JCMT datum, one of the motivations in this paper for arguing for the presence of a jet, the overall fit to the broadband spectrum is striking, with important implications for the interpretation of hard X-ray spectra if correct.

\section{ACKNOWLEDGEMENTS}

We would like to thank the staff of the JCMT for approval and execution of these observations at short notice. The JCMT is operated by The Joint Astronomy Centre on behalf of the UK Particle Physics and Astronomy Research Council (PPARC), the Netherlands Organisation for Scientific Research and the National Research Council of Canada. MERLIN is operated as a National Facility by the University of Manchester at the Nuffield Radio Astronomy Laboratories, Jodrell Bank, on behalf of the Particle Physics and Astronomy Research Council (PPARC). operated by The Observatories on behalf of the PPARC. We thank the staff at MRAO for maintenance and operation of the RT, which is supported by the PPARC.

\section{REFERENCES}

Blandford R., Königl A., 1979, ApJ, 232, 34

Brocksopp C., Jonker P.G., Fender R.P., Groot P.J., van der Klis M.. Tingay S.J., 2000, MNRAS, in press, (astroph/0011145

Corbel S., Fender R.P., 2001, ApJ, in prep

Corbel S. et al., 2001, ApJ, submitted

Dhawan V., Mirabel I.F., Rodriguez L.F., 2000, ApJ, 543, 373

Dhawan V., Pooley G.G., Ogley R.N., Mirabel I.F., 2000, IAU Circ. 7395

Di Salvo T., Done C., Zycki P.T., Burderi L., Robba N.R., 2000, ApJ, submitted

Dubus G., Kim R.S.J., Menou K., Szkody P., Bowen D.V., ApJ, submitted, (astro-ph/0009148)

Fender R.P., 2000a, Black hole states and radio jet formation, L. Kaper, E.P.J. van den Heuvel, P.A. Woudt (Eds), 'Black holes in binaries and galactic nuclei', ESO workshop, SpringerVerlag, in press (astro-ph/9911176)

Fender R.P., 2000b, MNRAS, in press, (astro-ph/0008447)

Fender R.P., Pooley G.G., 1998, MNRAS, 300, 573

Fender R.P., Pooley G.G., 2000, MNRAS, 318, L1

Fender R.P., Bell Burnell S.J., Williams P.M., Webster A.S., 1996, MNRAS, 283, 798

Garcia M., Brown W., Pahre M., McClintock J., Callanan P., Garnavich P., 2000, IAU Circ. 7392

Markoff S. Falcke H.Fender R., 2000, ApJ Lett, submitted, (astro-ph/0010560)

Hynes R.I., Mauche C.W., Haswell C.A., Shrader C.A., Cui W., Chaty S., 2000, ApJ, 539, L37

Merloni A., Di Matteo T., Fabian A.C., 2000, MNRAS, 318, L15

Patterson J., 2000, IAU Circ. 7412 
Pooley G.G., Fender R.P., 1997, MNRAS, 292, 925

Pooley G.G., Waldram E.M., 2000, IAU Circ. 7390

Remillard R., Morgan E., Smith D., Smith E., 2000, IAU Circ. 7389

Stirling A., Spencer R., Garrett M., 1998, New Astronomy Reviews, 42,657

Stirling A.M., Spencer R.E., de la Force C.J., Garrett M., Fender R.P., 2000, MNRAS, in prep

Taranova O., Shenavrin V., 2000, IAU Circ. 7407

Uemura M., Kato T., Yamaoka H., 2000a, IAU Circ. 7390

Uemura M. et al., 2000, PASJ, 52, L15

Wilson C.A., McCollough M.L., 2000, IAU Circ. 7390

Wood K.S. et al., 2000, ApJ, 544, L45 